\title{
Non-market Factors Affecting Price Volatility of Traditional Chinese Medicine Resources and Governance Mechanisms to Address Them
}

\author{
Shen Yuan ${ }^{1}$, Shen Jun-long ${ }^{2, ~ *, ~ Z h a n g ~ H a i-b o ' ~}{ }^{2}$, Yuan Pan $^{2}$ \\ ${ }^{1}$ School of Applied Mathematics, Nanjing University of Finance \& Economics, Nanjing, China \\ ${ }^{2}$ Management College of Economics and Trade, Nanjing University of Chinese Medicine, Nanjing, China
}

Email address:

ocsiban@126.com (Shen Yuan), jlshen2005@126.com (Shen Jun-long)

${ }^{*}$ Corresponding author

\section{To cite this article:}

Shen Yuan, Shen Jun-long, Zhang Hai-bo, Yuan Pan. Non-market Factors Affecting Price Volatility of Traditional Chinese Medicine Resources and Governance Mechanisms to Address Them. Science Journal of Public Health. Vol. 4, No. 3, 2016, pp. 183-190. doi: $10.11648 /$ j.sjph.20160403.15

Received: March 26, 2016; Accepted: April 28, 2016; Published: May 4, 2016

\begin{abstract}
In recent years, prices of traditional Chinese medicine (TCM) resources have undergone abnormal fluctuations due to intense volatility. It is believed that there are special reasons behind these fluctuations. The prices of TCM resources should be set with regard to market equilibrium. A survey of nearly 15 years' worth of data shows that non-market factors, namely social factors, are the main reasons for the price fluctuations of Chinese herbal medicines. This paper explores these factors and suggests reasonable control strategies to help stabilize the prices of TCM resources.
\end{abstract}

Keywords: Traditional Chinese Medicine, Price Fluctuation, Non-market Factors, Governance Mechanisms

\section{Introduction}

While traditional Chinese medicine (TCM) is recognized as the mainstay of Chinese medicine, the efficacy of Chinese medicine depends largely on the quality of herbal medicines used. TCM has been popular for many years because its resources are natural and it causes fewer adverse clinical reactions when used for disease prevention and treatment. At the same time, the prices of TCM resources directly affect the level of TCM's accessibility to patients. Thus, high quality and favorable prices are important factors affecting the use of TCM. TCM resources are divided into plant resources, animal resources, and mineral resources. The quality and efficacy of TCM have been clinically proven over thousands of years. Market price fluctuations are directly related to patients' medication needs. However, it is also important to study how the quality and price of genuine TCM are affected by non-market factors. The protection of natural TCM resources via prudent management strategies can help the Chinese government to control the social factors affecting TCM resource prices. Such strategies would help preserve TCM and assist its balanced and sustainable development in the future.

\section{Price Fluctuations of TCM Resources}

The laws of economics dictate that the ratio of production and supplementation of goods is governed by social consumption and demand, which are inversely proportional to the price. However, although the sales of Chinese herbal medicines have been increasing, so have their prices. This is contrary to the law of supply and demand. The market for Chinese herbal medicines is large and considerable research has been conducted with regard to the markets selling these items, such as Bozhou of Anhui Province, Hehuachi of Sichuan Province, Qingping of Guangdong Province, and so forth. Concentrating on quality specifications and graded divisions in Chinese herbal medicines in professional markets, such studies have followed the average prices of nearly 200 kinds of Chinese herbal medicines for about 15 years. However, these studies have focused only a few typical and genuine medicinal materials, such as White Peony Root, Honeysuckle, Angelica, Polyporus, Gastrodin, Aspongopus, Saposhnicovia Divaricata, acorus tatarinowii, Panax Notoginseng, hirudo, cantharides, tabanus bivittatus mats, and Cordyceps, while analyzing the 
fluctuations in prices. Most of these medicines showed considerable price differences; for example, the differences in the prices of White Peony Root and Cordyceps could range from 20 yuan $/ \mathrm{kg}$ to $7000 \mathrm{yuan} / \mathrm{kg}$, respectively. Thus, an analysis of such wide-ranging price fluctuations on the same graph would not be appropriate. Therefore, we tackle this problem by using the average price levels. We divide 13 herbs into three groups. Group 1 (the "general" group) includes White Peony Root (EC goods), Honeysuckle (EC goods), Angelica (grass), Polyporus (EC goods), Gastrodin (mixed), Aspongopus (EC goods), wind (EC goods), and Shi Chang Pu (EC goods). Group 2 includes slightly more expensive herbs such as Panax Notoginseng (120), leeches (water), Cantharidin (EC goods), and specific insects (Datong). Group 3 includes the most expensive herbs included in this study, namely, Cordyceps (3 500). Using a line chart, we plot the average price fluctuations of these traditional and genuine Chinese herbs over the past 15 years (Fig. 1).
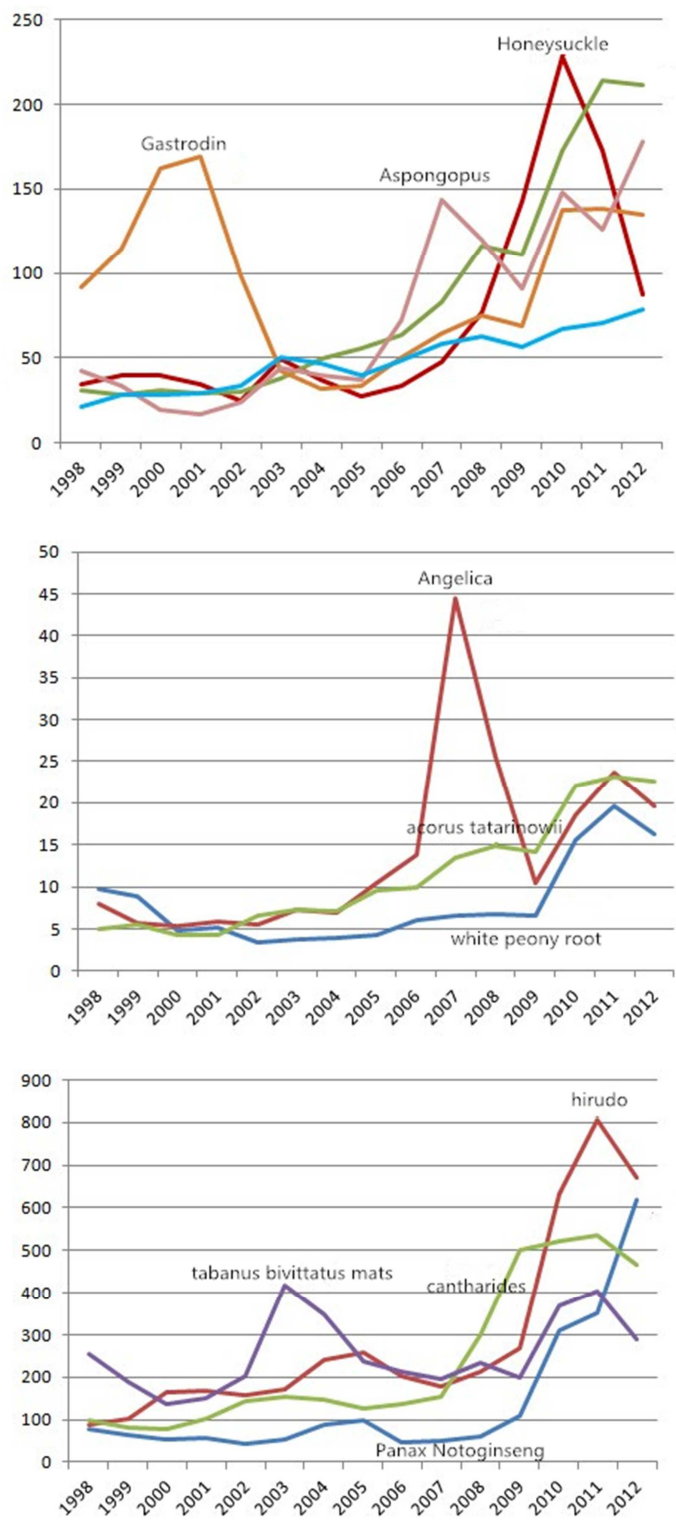

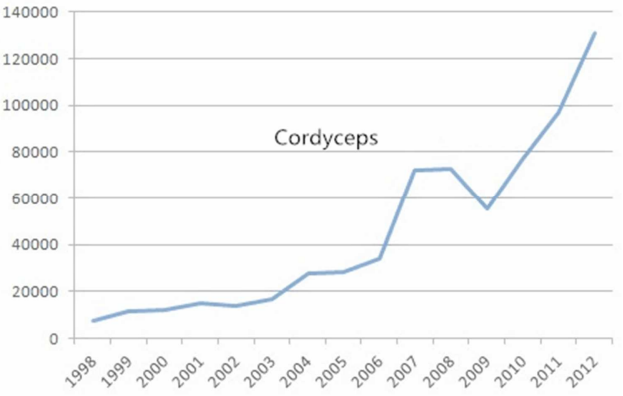

Fig. 1. Price fluctuations of select traditional and genuine Chinese herbs over the past 15 years.

Figure 1 shows relatively large price fluctuations for Honeysuckle, Polyporus, Gastrodin, Aspongopus, and Angelica. The prices of leeches and Panax Notoginseng are also quite volatile. Moreover, Cordyceps, which is very expensive, shows particularly high price volatility; its price appeared to decline for some time before soaring again in 2008 and 2009. These abnormal fluctuations in prices are superficially linked to market supply and demand factors; in reality, they are caused by certain social factors. This paper studies some of these factors.

\section{Analysis of Non-market Factors Affecting Price Volatility of TCM Resources}

\subsection{The Impact of Medicinal Herb Growers'Social Psychological Expectations}

Currently, most TCM resources are highly dependent on growers/farmers who acquire, plant, or breed them. The level of organization at such scales of production is low. There is a high degree of uncertainty and farmers are forced to accept the market price. In turn, they tend to determine their next output based on the current market price [1]. Thus, the growth cycles of traditional and genuine Chinese herbs tend to be largely unplanned, and the farmers are unable to time planting or breeding cycles in the most efficient manner possible. With regard to the life cycles of TCM resources (namely, geoherbs having commodity characteristics), the elasticity of supply is greater than that of demand. Therefore, this is the result of internal cause of price fluctuation divergent spider mesh [2].

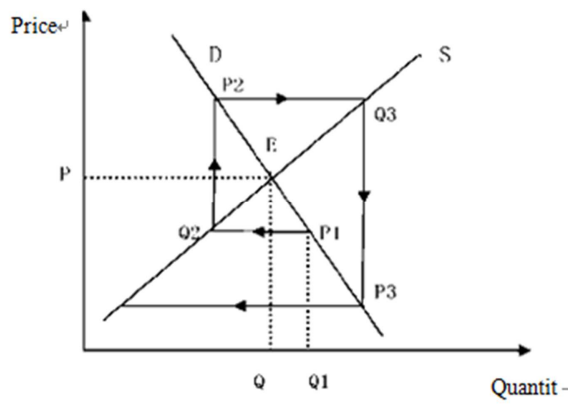

Fig. 2. Divergent cobweb model explaining the price fluctuations of genuine TCM resources. 
For instance, White Peony Root is a perennial herb with a growth period of about 5 years. The growth period of Panax Notoginseng, Platycodon Grandiflorum, and Cortex Phellodendri is 3 to 7 years, 3 to 5 years, and 10 years, respectively. To ensure the quality of Chinese medicinal materials, the herbs must be harvested within the correct period of time. Growers plan their planting and breeding schedules based on the current prices of the materials in the market. The emergence of the global flu epidemic in the second half of 2009 intensified the gap between supply and demand, for example, by sending the prices of White Peony Root soaring. Many growers attempted to judge the future price of the herb in the market, leading to irrational behavior. They started harvesting the root too early, when it was immature. Thus, its yield rose significantly in 2010, but given the great demand for the root, prices did not decline. In addition, there was much speculative hoarding of supplies, causing the price of White Peony Root to rise continually. Thus, its price continued to rise, further fuelling the enthusiasm of the growers, who continued to plant vast quantities of White Peony Root to increase yields. As Figure 2 shows, a large number of speculators sold White Peony Root in 2011 owing to its price volatility (sharp decline). This practice follows the typical characteristics of a divergent cobweb (Figure 2).

Because Chinese herbal medicine resources are mainly produced by small-scale agricultural farmers as a side business, we can observe characteristics of obvious periodicity. When the price of a TCM resource is too low (P1), Chinese producers tend to reduce the scale of production (Q2), leading to short supply in the next phase and pushing prices up sharply (P2). When the price of a TCM resource is high (P2), it often leads to a huge surge in production (Q3), which typically exceeds actual market demand. Since the price of the resource is determined by future production and demand, there is a sharp fall in its price (P3).

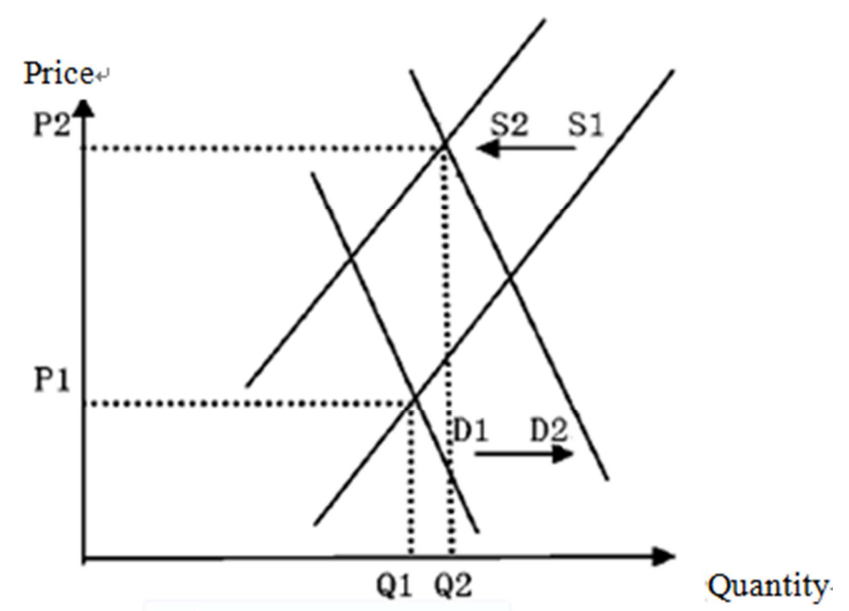

Fig. 3. Price booms of genuine TCM resources led by hot money hoarding.

\subsection{Distortion of the Market by Human Intervention/Hot Money Speculation}

When the market operates in the normal mode, real supply and demand affect the prices of commodities. The price fluctuations occur around a reasonable value, in accordance with the law of value of a commodity in the economy. From the perspectives of a longer period and the whole society, a commodity's price and value deviate both positively and negatively, canceling each other out. Thus, the overall commodity price and value remain more or less the same. In reality though, market failure phenomena, such as hot money hoarding speculation, can produce an amplified distortion of the supply and demand relationship [1].

As Figure 1 shows, while the price of Angelica was slightly higher than average in 1998, on the whole, its price has been relatively stable in the 20th century (average price of 5 to 7 yuan $/ \mathrm{kg}$ ). With the development of a new rural cooperative medical system, including medical insurance for urban residents and medical security for workers, the use of Angelica has recorded a slow rising trend. Until 2007, the price of Angelica showed a sudden steep rise, with the average price reaching thrice the value in 2006 . The price of Chinese Angelica soared because of hot money speculation rather than real changes in market supply and demand. Consider Minxian county as an example, where the price of Angelica doubled at the beginning of 2007. From May to mid-August year, the price of Angelica reduced, as a large number of speculative traders bought and hoarded the plant, anticipating price increases. Thus, Angelica became scarce, and in 2008, its market sales price reached nearly 45 yuan $/ \mathrm{kg}$. Speculators took the opportunity to dump Angelica, resulting in a sharp decline in its price. Thus, when many speculators resort to hot money speculation, they hoard large quantities of Chinese medicinal materials, leading to false increase in market demand [3]; this is depicted by the shifting of the demand curve to the right, from D1 to D2. At the same time, hoarding medicinal resources reduces their effective supply in the market, as seen by the shift in the supply curve to the left (from S1 to S2), and the market price rises significantly (from $\mathrm{P} 1$ to $\mathrm{P} 2$ ), creating serious fluctuations in the value of commodity prices (Figure 3 ).

\subsection{Unexpected Risk Factors, as Explained Below, Can Also Cause Price Changes}

\subsubsection{Abrupt and Natural Climate Disasters}

The southwestern region of China is an important origin of TCM resources. Chongqing alone supplies more than 4300 Chinese herbal medicine resources, while the Yunnan Province is home to 4758 medicinal plants, 260 species of medicinal animals, and 32 kinds of mineral medicine. In recent years, natural disasters have occurred frequently in the southwest of China, and the area affected by and intensity of such disasters has increased. Natural disasters have caused price rises of the geoherbs grown in these zones.

Consider Panax Notoginseng grown in Wenshanzhou, for example. As Figure 1 shows, since 2008, the price of Panax Notoginseng has been rising. By 2012, it averaged at 120 to 600 yuan $/ \mathrm{kg}$, about 10 times the price of Panax Notoginseng in 2008. There are unique reasons behind these skyrocketing prices. About $97 \%$ of China's Panax Notoginseng is produced 
in Wenshanzhou and HongHezhou in Yunnan Province. In 2008, Wenshan suffered frost damage, while Yunnan Province faced drought in 2009. The maturity period of Panax Notoginseng is 3 to 7 years. The four years of consecutive drought seriously affected the growth of the roots and herbs, resulting in declining yields of Panax Notoginseng. The demand for it is increasing, however, and in accordance with the law of supply and demand, the prices of Panax Notoginseng have soared in the last four years.

\subsubsection{Epidemic Outbreaks}

As can be seen from Figure 1, the price fluctuation of Honeysuckle is closely related to the outbreak of the SARS epidemic in China. The average price of Honeysuckle peaked in 2003 and 2010. The SARS outbreak in 2003 led to a surge in demand for genuine medicinal materials having detoxification and anti-bacterial qualities, as consumers were keen to improve their immunity. Thus, the price of this resource rose to about 50 yuan $/ \mathrm{kg}$. After the SARS outbreak was effectively controlled, the price of Honeysuckle gradually dropped to 20 yuan $/ \mathrm{kg}$. The low price reduced market supply. In 2005, China experienced an outbreak of avian flu, and the detoxifying and antibacterial effects of Honeysuckle were once again in much demand. Thus, its price increased quickly, reaching 40 to 50 yuan $/ \mathrm{kg}$. Then, in 2009, the spread of H1N1 flu around the world once again increased the demand for this resource, resulting in short supply, which drove up its price to 200 yuan $/ \mathrm{kg}$. Indeed, 2009 and 2010 saw the most rapid increase in the price of Honeysuckle. Thus, an epidemic outbreak can also be a big contributing factor in the price fluctuations of Chinese herbal medicines.

\subsection{The Influence of Economic Development and Improvement in Living Standards}

With the development of Chinese economy, people's health awareness and living standards have gradually improved. Thus, they tend to pay more attention to their health, leading to a rapid increase in the demand for precious TCM resources. Some consumers favor the use of Cordyceps Sinensis. Because Cordyceps is considered to detoxify the liver, lungs, and kidneys, besides improving yin and immunity function, it is known as the "first grass," "golden grass," or "fairy grass." In fact, Cordyceps, ginseng, and Pilose Antler are called the "three treasures of TCM." Therefore, Cordyceps is much sought after as a "star" tonic, especially in the high-end consumer group. The rising demand for Cordyceps can be seen in its high wholesale price of about 140,000 yuan $/ \mathrm{kg}$.

\subsection{The Impact of National Policies on TCM Resource Demand}

Around 2009, China adopted the Keynesian policy, stimulating domestic fiscal and monetary policies, increasing prices, and inevitably, inflation. Chinese herbal medicinal resources are cultivated via small-scale agriculture. Inflation affects the prices of grain, oil, sugar, cotton, and other agricultural commodities. An increase in these prices directly impacts the Chinese medicinal resources, leading to higher prices of TCM resources. Figure 1 shows that the prices of many Chinese herbal medicines soared in 2010, and this can be attributed partly to widespread inflation.

National macro-policy changes can also affect the prices of Chinese herbal medicines. In recent years, China has promulgated the protection of rare and endangered animals and plants; sustainable development; erosion control; protection of natural forests, pastures, and grazing grass; and the agricultural sector through structural adjustment policies. Such major decisions have been taken to protect TCM resources and yields, by bringing about a reduction in resource prices and controlling price fluctuations [4]. Moreover, the continuous improvement in the Chinese medical system promotes medical security. For instance, the inclusion of Chinese herbal medicines in the national basic drug list can allow medical institutions to prescribe the same to patients, increasing the market demand for TCM resources. To establish the role TCM within the medical system, it is necessary to transform and upgrade TCM resources by developing a TCM resource supply chain. As Figure 1 shows, with regard to Gastrodia elata Blume and Polyporus, abnormal fluctuations in prices can be attributed to the above-mentioned policies, which reduced their supply despite increased demand.

\section{The Social Mechanisms Controlling Price Fluctuations of TCM Resources}

\subsection{Technological Innovation of TCM Resources}

China's economy is facing major changes by way of development. In particular, enterprise development must place the pursuit of quality and efficiency at its core. China's supply-side reforms require the TCM resources industry to be innovative as well as eco-friendly (sustainable). Thus, there is an urgent need to speed up the development of the TCM resources industry.

The quality of TCM resources should be such that the desired clinical effects must be achieved each time. However, in reality, clinical experiences with TCM show that the clinical efficacy of Chinese herbal medicines varies in different regions; indeed, for thousands of years, much emphasis has been put on using genuine TCM resources to achieve the required clinical efficacy. For example, certain TCM resources, like Angelica from Minxian county; Ginseng from Dunhua, Fushun, and Changbai; Panax Notoginseng from Wenchuan and Honghe; Fritillaria grown in Songpan and Kangding; Curcuma from Guandong province; and Dendrobium from Huoshan, have always been recognized as genuine materials by TCM clinics.

Notably, the growth periods of Chinese medicinal herbs are well known. These growth periods are affected by the growing practices and climatic conditions of the region the resource is grown in. Thus, these factors should be considered while ensuring the quality, and hence clinical efficacy, of the herbs 
[5]. If any other wild resources are introduced and domesticated, the growth period of that species of Chinese medicinal herb is likely to change. TCM resources are scarce to begin with owing to their cyclical growth patterns, which cannot change with social demand. Moreover, the stock and regeneration capacity of wild TCM resources are limited. Because of the influence of the natural climate of the region the resources are grown in, Chinese herbal medicines cannot be planted in all regions. As their growth periods are specific and limited, most farmers are prone to use chemical fertilizers and pesticides during the cropping phase, hoping that the harvested crop will provide good yields. Unfortunately, some farmers tend to harvest the herbs before their maturity period is reached and the use of fertilizer and pesticide residues degrade the quality of the TCM product.

The quality of TCM resources is also affected by the land use cycle. The area suitable for planting some Chinese herbal medicines is limited, the fertility of and trace elements in such land are finite, and after harvesting, the land used to grow some Chinese herbal medicinal crops must be left fallow. Henan province is also called the origin of "Four Huai Medicines." Dual-purpose crops like yam are used as medicine as well as food. In recent years, the market demand for yam has increased, which has led to an increase in the land area used for yam cultivation. However, most farmers refuse to leave the land fallow after harvesting the yam crop, resulting in depletion of trace elements and affecting its fertility negatively. Consider the example of Wenshanzhou in Yunnan province, where wild Panax is planted. Panax Notoginseng has particular soil requirements. The land used to grow the crop must be left fallow for at least 10 years before replanting because the cultivation of Panax Notoginseng is known to harbor pestilential bacteria and viruses in the land, which are adverse to the new Panax Notoginseng crop [6]. Thus, repeated planting may cause root rot diseases to occur, seriously influencing the yield and quality of the crop. Indeed, the issues caused by continuous Notoginseng cropping have been studied in much detail by the Panax Notoginseng industry. Addressing this problem is crucial to prevent the degradation and depletion of the already scarce land resources capable of growing this crop. The price of such land has already started rising, and the added costs cultivation are passed on to the consumer via the increased price of medicinal herbs. Thus, the manner of land use has also led to abnormal fluctuations in the prices of genuine Chinese medicinal materials.

The regeneration ability of wild TCM resources is yet another factor affecting the quality and yield of Chinese medicinal materials. The quality and regeneration ability of genuine TCM materials have long been recognized as key supply constraint factors. For thousands of years, TCM resources have been viewed as natural products in that they do not require much human labor for growth. However, with the development of planting and breeding technologies and biotechnology, farmers have been able to increase the supply of some TCM resources by artificial cultivation. Various restrictions prevent farmers from using advances in artificial breeding or cultivation to increase the yields of all varieties of Chinese herbal medicines. As per the Third Chinese Materia Medica Resource Inventory, $80 \%$ of medicinal herb varieties in China continue to be sourced from the wild (i.e., without artificial breeding or cultivation). Saposhnicovia Divaricata is commonly used in the domestic Chinese herbal medicines market. It is one of the three wild herbs that enjoys regulatory protection and is considered to be an irreplaceable species. The varieties produced in three northeastern provinces and Inner Mongolia are different. Its production is falling, and its continual short supply has led to an increase in its price on an annual basis. Given its excessive exploitation and induced resource depletion, the species is regarded as endangered.

If the Chinese medicinal industry is to participate in the new economic order, it must first guarantee quality. This requires an innovative technology system, which can integrate traditional techniques and modern technology. Because traditional experience has its limitations, namely the lack of objective chemical indicators, modern technology cannot yet determine whether certain chemical components of Chinese medicinal herbs are clinically effective. Moreover, because each Chinese herbal medicine is used for multiple purposes and one medication can complement another, it is difficult to ascribe the effects of the medication to one component alone. In ancient China, the quality of Chinese herbal medicines was judged according to long-term practical knowledge and experiences of the doctors at the time. Thus, the morphological features discrimination ginseng growth years with a number of $\mathrm{Lu}$ tou petiole scars, wild Gastrodia elata parrot mouth, cinnabar point to identify, the judgment of wild gastrodin is its parrot-shaped mouth and spot of oil cavity. With regard to texture and hardness as identification parameters, for example, the surface of Angelica is yellowish-brown or sepia. It has fine wrinkles and longitudinal, horizontal, and convex lenticel-like scars. The surface of Semen Arecae is gray white and has brown-colored marble veins. The cross-section of Polygonum Multiflorum shows tertiary vascular bundles shaped in a brocade pattern. With regard to smell as a parameter of identification, Gastrodia smells like horse urine and tastes a little sweet, while the smell of musk can last for a long time. Taste has also been used to identify herbs. For example, Angelica Sinensis and Angelica Pubescens look similar, although Angelica Sinensis tastes sweet and bitter while Angelica Pubescens tastes bitter and spicy. The previously sweet taste of Calculus Bovis changes afterwards to bitter and a cool feeling can be experienced at the base of the tongue [7]. However, while these parameters can be used to determine the quality of Chinese medicinal materials, they can only be identified by practically experienced workers in the industry.

With the development of science and technology, geoherb quality evaluation is benefitting from some modern research methods, such as TCM fingerprinting and the Dao-Di Index (DDI). TCM fingerprint analysis is based on the characteristics of unique populations within species. Finding similarities in the same chemical composition of a medicinal herb population helps scientists to identify its uniqueness. 
This is analogous to the fingerprinting concept used in forensic science. TCM fingerprinting can fully reflect the chemical compositions contained in the herbs, thus assessing the quality of the medicine's components [8]. Xiao Xiao-he et al. [9] comprehensively studied the history and ecological suitability of herb-producing areas, commercial specifications and grades of TCM resources, and growth rates of the main components. They weighted the biopotency/toxic potencies of the resources to construct a comprehensive index providing quantitative indicators and standards for quality evaluation and control of genuine Chinese medicinal herbs. The State Department or industry organizations can help by organizing experts toward building a common technical platform for geoherb quality assessment. By combining the above-mentioned techniques, it will be possible to establish a quality standard/system for genuine TCM resources, so as to ensure their quality and clinical efficacy.

\subsection{Protection of Chinese Natural Medicinal Resources, and Improvement in Resource Efficiency and System Innovation}

\subsubsection{Establishment of Development Goals for TCM Resources}

Traditionally, the development and resource efficiency of TCM resources has been constrained. China's ancient philosophers used natural resources and wrote extensively of their benefits as medicine. The Book of Changes, for example, explains the concept of "Yin and Yang," namely the need for balance and order, while high officials in ancient China referred to "Huanglaoism," the concept about the mutual generation and restriction of the five elements and their functional cycles. The ancient Chinese medical text of Huangdi Neijing stresses upon the importance of harmony between man and nature, as does Tao Te Ching. Qimin Yaoshu, the most completely preserved of the ancient Chinese agricultural texts, notes that obedience to Heaven means adapting to nature, while the Chinese philosopher Mencius emphasized the protection of natural resources and their appropriate use. These views point to the need to transform and develop the Chinese TCM industry into one that requires low input but performs efficiently, while simultaneously protecting resources and using them sustainably.

With the continuous development of human and technological progress, China's total population has increased dramatically in modern times. Natural TCM resources have been unable to meet the resulting social needs, and some areas have been exploiting these resources extensively, leading to their degradation and depletion. As noted previously, the increasing frequency of natural disasters exacerbates the situation. China has recognized the threats posed by these issues, and since 2012, China's economic development has entered a new normal state. At the 18th Party Congress, the Central Committee of the Communist Party of China directed that the country's future development will follow the principles of a green economy.

\subsubsection{Establishment of a Public Information Service System for TCM Resources}

The Fourth National Census of TCM resources provides much-needed and updated information pertaining to natural TCM resources. There is a need to plan timely releases of pertinent information, improve transparency with regard to the genuineness of medicinal materials, and provide market information to growers so that they may reasonably plan planting and harvesting. Doing so would help strike a balance between supply and demand. Moreover, the establishment of a public trading platform could promote transparency, increase sales/traded volumes, enable planned stocking of material resources, prevent speculation, and strengthen monitoring in the TCM market. Encouraging online contract transactions will reduce the number of middlemen, speed up transactions in the market, and reduce transaction costs. This would also improve the efficiency of resource utilization and help control prices of genuine TCM products.

\subsubsection{Using Two-Dimensional Code Technology to Establish Traceability and Accountability}

Quality and price management of TCM resources can be ensured through various avenues. The State Food and Drug Administration can mandate that producers disclose detailed information such as the source of the medicine, its morphological identification, content determination, processing method, and so on. Modern technology, such as the two-dimensional code technology, can be used to establish traceability and improve accountability. For example, Sichuan province has launched the "TCM traceability system," wherein scanning the two-dimensional bar code on the medicine container activates a sensor display that provides details on various parameters of the medicinal materials. Thus, the medicinal materials can not only be identified correctly but the technology may also be used as an effective measure to standardize the quality of medicinal herbs and manage non-market factors influencing abnormal price fluctuations of TCM resources.

\subsubsection{Establish an Appropriate Protection and Utilization Policy for TCM Resources}

It is crucial to formulate a suitable policy for the protection and utilization of TCM resources. Traditionally, people have not attached importance to the conservation and sustainable use of genuine Chinese medicine resources, resulting in the depletion of some precious medicinal plants. A supply shortage will inevitably lead to higher prices. Today, people recognize that unsustainable use of wild Chinese medicinal herbs needs to be controlled. Therefore, countries need to develop effective policies for the protection and rational use of geoherbs. To this end, the state may establish gene banks for genuine medicinal materials. It can promote germplasm collection and conservation, and strengthen artificial cultivation practices to ensure improved protection of genuine medicinal materials [10]. Notably, standardization is very important to be able to grow genuine medicinal materials of consistent quality [11]. Currently, national gene banks allow 
long-term germplasm storage periods at temperatures of $-18 \pm$ $1^{\circ} \mathrm{C}$. These banks also help conserve crops that reproduce asexually, recalcitrant seed crops, and other special germplasm resources. To maximize resource efficiency, the government should extend support to research into other vital aspects such as developing multiple uses for geoherbs used in traditional medicine, determining whether other parts of the plant also have medicinal value, studying optimal harvest times, and so on. Such initiatives will eventually result in economic, social, and ecological benefits.

By itself, each Chinese herbal medicine is a gene bank of sorts as it contains numerous substances of medicinal value. Thus, clinically, many TCM resources often have a variety of disease treatment and prevention functions. The development and utilization of TCM resources via modern technology should not only concentrate on their use as medication but also as reservoirs for pharmaceutical materials. China, therefore, needs to develop specific policies to promote the protection of wild medicinal resources, their artificial domestication, and habitat preservation so that ecological balance can be maintained. The biodiversity of TCM resources ensures that they can have multiple uses, namely as medicines and health care products, food, and additives in other products, as well as in the pharmaceuticals, agriculture, forestry, horticulture, and environmental protection industry. Therefore, the government should formulate policies to promote the development of a circular economy.

It is also important to ensure intergenerational equity in the use of TCM resources. Economic development, improvement in people's living standard and health awareness, and improved information provision via the Internet have increased the demand for TCM resources. Ironically, these very factors have also shed light on their limited resources and inconsistent performance. Unfortunately, some pharmaceutical companies and private organizations only pursue immediate short-term benefits, ignoring the long-term need for sustainable development and limited regenerative capacity of TCM resources. When the number of individuals in the wild natural population falls to a certain level, there is a real risk of loss of some medicinal species, which would have negative consequences on TCM resources. Thus, to avoid future generations from losing such species to extinction and to promote intergenerational equity, the government must attempt to establish a much-needed balance between the protection of TCM resources and their utilization.

\subsubsection{Establishing a Reserve System for Market Cycles}

The demand of TCM resources is strong but prone to market vagaries. In order to reduce the adverse effects of sudden changes in Chinese and international market demand, there is a need to guarantee an adequate supply of medicines. In other words, it is necessary to balance supply and demand, and ensure stable prices. This can be achieved by establishing a reserve system for market cycles. On the one hand, the state should serve as the main repository of TCM resources. On the other hand, it should actively encourage private capital toward TCM resource preservation and supplement it when needed via grants. First, the government should enter into cooperation agreements with private pharmaceutical companies and large Chinese herbal medicine businesses to identify reserve TCM resource units and ensure the uninterrupted supply of quality Chinese herbal medicines in unexpected situations. Second, the government should also establish an efficient reserve dispatch system for Chinese herbal medicines to provide a channel for their timely supply. Third, the creation of a national network or directory can provide important information about and promote cooperation among Chinese herbal medicine manufacturers, growers, and other related personnel. Finally, a national organization comprising personnel with scientific and traditional knowledge about TCM is needed to conduct research on artificial cultivation and breeding technologies, and to strengthen international technical exchange and cooperation in order to promote the sustainable development of TCM resources.

\section{Conclusion}

TCM resources are strategic, economic, and irreplaceable resources vital for the continued development of the Chinese pharmaceutical industry. Their protection and efficient utilization are crucial. Excessive use of TCM resources will inevitably result in ecological decline via grassland degradation, soil erosion, and loss of biodiversity, leading to undesirable price fluctuations. For these reasons, it is crucial to consider the maximum carrying capacity of the ecological environment, speed of resource regeneration, and supply. Continuous technical innovation and careful planning can improve the utilization efficiency of TCM resources. The Chinese government should therefore institute appropriate policy mechanisms to safeguard these resources and simultaneously reap the economic, ecological, and social benefits they provide.

\section{Acknowledgments}

The study was sponsored by Agreement project sponsored by the US WYETH: "Study on the hierarchical quality classification and the quality price correlation of Chinese Herbal Pieces". We thank Prof Shen junlong for contributions to study design, study coordinatio and study management; Zhang haibo for market research, data collection and data analysis; Yuan pan for checking the Article's language and style.

\section{References}

[1] Chen Hui, Wang Huo-wang. Reflections on Chinese herbal medicines price control regulation policy [J]. Price supervision and inspection in China. 2013(1): 42-44.

[2] Zhao Huan, Qu Kai-yue, Li You-xin. Analysis and prevention on market risk of Chinese medicinal materials based on "cobweb model" [J]. Chinese Traditional and Herbal Drugs, 2013, 44(2): 238-240.

[3] Wang Yong-jie. Minxian county angelica price change Reasons and Countermeasure $[\mathrm{J}]$. Agricultural Science and Technology. 2008, 01: 44. 
[4] Ren Chang-qiu. Causes and Effects of Chinese herbal medicines price changes [J]. People's Tribune. 2011(2): 142-143.

[5] Wan renfu, Xu weiya, Wang shaojun, Ouyang hui, Li yujiao, Gong qianfeng, Yang shilin. Discussion on the Strategy for Development of Authentic Medicinal Herbs [J]. China Pharmacy 2007. 18(9): 641-643.

[6] Sun xue-ting, Li lei, Long guang-qiang, Zhang guang-hui, Meng zhen-gui, Yang sheng-chao, Chen jun-wen, The progress and prospect on consecutive monoculture problems of Panax notoginseng. [J] Chinese Journal of Ecology 2015, 34(3): 885893

[7] $\mathrm{Hu}$ fang, A brief discussion on the traditional methods of identifying traditional Chinese Medicine $[\mathrm{J}]$ Lishizhen Medicineand Materia Medica Research. 2006. 17(10).
[8] Li Qiang, Du Si-miao, Zhang Zhong-liang, Lv Chun-ming, Zhou Yong-quan, Zhao Yan, Zhang Ning. Progress in fingerprint technology on Chinese materia medica and prospect of its future development $\lceil\mathrm{J}\rceil$. Chinese Traditional and Herbal Drugs, 2013, 44(22): 3095-3104.

[9] Xiao Xiao-he, Wang Jia-bo, Yan Dan, Lv yang. Establishment of Dao-Di index and its significance in quality control and rational usage of Chinese medicine [J]. China Journal of Chinese Materia Medica, 2012, 37(11): 1513-1516.

[10] Zhao Yong-sheng, Zhou Ya-xing, He Wei, Zha Yun-sheng. Protection and exploitation of rare and endangered medicinal materials in $\mathrm{Nu}$ Jiang Zhou [J]. Chinese Journal of Ethnomedicine and Ethnopharmacy. 2005, 14(6): 350-354.

[11] Zhang Wen-sheng, Wang Li-yang. Geoherbs correlation with environmental factors [A]. China research and promotion of TCM, 2003. 